\title{
Influence of melt convection on the dynamics and capture inclusions for growing oxide crystals by HDC
}

\author{
S.V.Naydenov, S.V.Nizhankovskiy, A.V.Tan'ko, \\ L.A.Grin', V.V.Baranov \\ Institute for Single Crystals, STC "Institute for Single Crystals" National \\ Academy of Sciences of Ukraine, \\ 60 Lenin Ave., 61001 Kharkiv, Ukraine
}

\section{Received March 6, 2015}

\begin{abstract}
The main properties of motion of gas-vapor bubbles and impurity inclusions in hydrodynamic flows are considered. It is founded the criteria for the formation of the defective areas for crystal growth of oxide crystals by horizontal directional solidification which is depended on the hydrodynamic and geometrical parameters of the melt convection.

Keywords: crystal growth, gas-vapor bubbles and impurity inclusions, hydrodynamic flows, defective areas, horizontal directional solidification, convection structures.

Рассмотрены основные закономерности движения парогазовых пузырей и примесных включений в гидродинамических потоках. Найдены критерии образования дефектных зон при выращивании оксидных кристаллов методом горизонтальной направленной кристаллизации в зависимости от гидродинамических и геометрических параметров конвекции расплава.
\end{abstract}

Вплив конвекції розплаву на динаміку і захоплення включень при вирощуванні оксидних кристалів методом ГНК. С.В.Найдьонов, С.В.Нижанківський, А.В.Танько, Л.А.Гринь, В.В.Баранов.

Розглянуто основні закономірності руху парогазових бульбашок та домішкових включень у гідродинамічних потоках. Знайдено критерії утворення дефектних зон при вирощуванні оксидних кристалів методом горизонтальної спрямованої кристалізації в залежності від гідродинамічних та геометричних параметрів конвекції розплаву.

\section{Introduction}

One of the major defects in crystals grown from a melt is various inclusions: vapor-gas bubbles and impurity phases (the material of the crucible, impurities, etc.) [1,2]. The mechanisms of their formation and capture by the crystallization front is deeply studied, but practical ways and methods for producing crystals without inclusions still insufficiently developed. This problem is particularly actual in growing crystals of refractory oxide (sapphire, yttrium aluminum garnet, etc.), suitable for the manufac- ture of large windows and laser elements of high optical quality. The main ways to reduce the probability of formation of inclusions in crystals are using of high-purity raw materials with low porosity, optimization of the growing atmosphere, temperature and convection conditions in the melt for effective removal of the gaseous components.

As shown by our experiments, and the results of mathematical modeling of the growing conditions of sapphire and garnet (YAG) by horizontal directional crystallization (HDC), formation of the two-vortex 


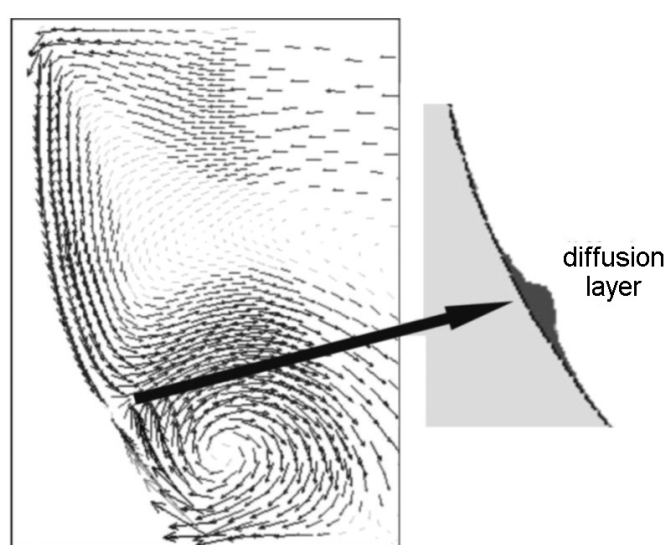

Fig. 1. Two-vortex motion of the melt and the diffusion layer near the crystallization front.

flow structure of the melt near the crystallization front is possible [3, 4]. This hydrodynamic structure leads to a local increase in thickness of diffusion layer, formation of stagnant zone in the area of contact of the vortices with solidification front (Fig. 1), accumulation of impurities and subsequent capture of the inclusions (vapor-gas bubbles, impurity phases). As a result the defective zone in the crystals having a domelike shape and is located along direction of the crystal growth ("longitudinal capture") (Fig. 2). Concentration of the defect region the inclusions in reaches values



In this article the physical laws of influence of the two-vortex melt convection on dynamics and capture of the inclusions during growing oxide crystals (sapphire, yttrium aluminum garnet) by HDC method are investigated.

\section{Hydrodynamic model of formation of the non-uniform distribution of inclusions in crystals}

Let's consider the theoretical model of segregation of inclusions by hydrodynamic vortex structures in the melt. We begin with analysis of the segregation of vaporgas bubbles. Profile of their distribution in the melt is formed as a result of complicated dynamics and growth of the bubbles. The bubbles growth in the melt can be considered independently from their dynamics, which depends on the total hydromechanical motion of the bubbles taking into account both the convective hydrodynamic fluxes and mechanical motion of the bubbles under influence of various hydrostatic forces a)

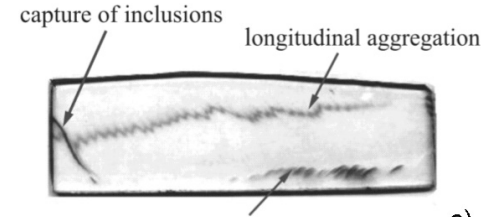

b)


Fig. 2. a) Longitudinal section of sapphire crystal containing inclusions, b) 3D-model of longitudinal aggregation in the crystal, c) cross section of sapphire crystal containing inclusions.

(gravity, buoyancy, force of viscous resistance). It is convenient to decompose the resulting dynamics of the bubbles on specific components of the motion and separately assess the role of each of them.

It should be distinguished three basic types of the bubbles motion: 1) hydrostatic bubble floating (the Archimedes force excluding viscosity); 2) hydrostatic floating with the forces of viscosity (the Stokes force); 3) hydrodynamic motion along the lines of the vortex flow. According to these motion types the characteristic times can be introduced: 1) the hydrostatic floating time; 2) the hydrostatic floating time taking into account of viscous resistance; 3 ) the time of removal under the action of hydrodynamic convective flows.

We assume a small effect of the change of hydrostatic pressure on the bubble size. This assumption is acceptable during crystal growth from the melt under pressure, e.g., under inert gas and small thickness of the melt, which common conditions for the HDC method, when the height of the melt does not exceed 50-60 $\mathrm{mm}$.

It is known [5] that the force of viscous resistance for motion of the bubble with diameter from $0.01 \mathrm{~cm}$ (up to $10 \mu \mathrm{m}$ ) is very well depicted by the Stokes law (directly proportional to speed). In the bubble size up to $100 \mu \mathrm{m}$ the Stokes law is also carried out with sufficient accuracy. Our studies have shown that size of the impurity inclusions in the longitudinal capture is about $1-10 \mu \mathrm{m}$ and captured gas bubbles is $10-50 \mu \mathrm{m}$ (Fig. 3). Therefore, to describe the viscous resistance 




Fig. 3. The bubbles in the horizontal grip. The size distribution of inclusions in the cross section of capture uniform.

in the melt the linear Stokes approximation can be used.

It is also known [5], that form of the gas bubbles remains nearly spherical (i.e., the bubbles behave like "solid balls"), when they floating or moving in a hydrodynamic flow at low or moderate Reynolds number of order of Re $\leq 700-800$. This is well performed in our case.

Let us estimate the number $\operatorname{Re}=u l / v$, where $u$ is characteristic speed of the object; $l-$ its characteristic size, namely the diameter $d$ of the bubble; $v=\eta / \rho-$ kinematic viscosity (measured in the Stokes, $\left.[S t]=\left[\mathrm{cm}^{2} \cdot \mathrm{s}^{-1}\right]\right)$, i.e. the ratio of dynamic viscosity $\eta$ and density of the fluid $\rho$. The characteristic rate of the bubble motion, as will be seen below, of the order of magnitude does not exceed $10 \mathrm{~cm} \cdot \mathrm{s}^{-1}$, that is of the order of the hydrodynamic flow characteristic speed. Characteristic diameter of the bubbles does not exceed a hundred microns, i.e., $d \leq 10^{-2} \mathrm{~cm}$. The melt density for sapphire or garnet: $\rho \sim(3 \div 4) \mathrm{g} \cdot \mathrm{cm}^{-3}$; the typical dynamic viscosity of the melt: $\eta=0.5 P$. Kinematic viscosity is of the order of $v \propto 10^{-1} \mathrm{~g} \cdot \mathrm{cm}^{-1} / 10^{1} \mathrm{~g} \cdot \mathrm{cm}^{-3} \propto 10^{-2} \mathrm{~cm}^{2} \cdot \mathrm{s}^{-1}$ $=10^{-2}$ St . Hence we get typical assessment: Re $\propto 10$. Therefore, the bubbles hydrodynamics is associated with the moderate Reynolds number, and conditions for preserving of their spherical shape are executed. The bubbles radius variation can occur due to mechanisms of the diffusion growth of the new-phase nuclei, coalescence, etc. Along with that the growth of the bubble itself can be considered regardless of their dynamics, as it follows from the assessments provided.
Let us estimate characteristic time $\tau_{\alpha}$ of the hydrostatic floating. We define the time of the bubble transfer from the bottom of the volume to its middle, that is, before the bubble reaches the intermediate layer between the macroscopic vortices. For volume height $H$ in the case of the two-vortex convection this layer is located approximately at $h^{*}=H / 2$. We assume that the bubble is transferred in the horizontal direction by fluid flow with a constant rate. In the vertical direction it is affected by the gravity force $F_{g}=m_{2} g=\rho_{2} V g$, directed vertically downward and by the Archimedes lift force $F_{A}=\rho_{1} V g$, directed vertically upward. Here $\rho_{1}$ and $\rho_{2}-$ densities of the liquid (melt) and the floating body (gas); $V$ - volume of the gas bubble; $g=9.8 \cdot 10^{-2} \mathrm{~cm} \cdot \mathrm{s}^{-2} \sim 10^{-1} \mathrm{~cm} \cdot \mathrm{s}^{-2}$ - acceleration of gravity.

The motion is uniformly accelerated with acceleration equal (in magnitude) to

$$
a=\frac{\left(F_{g}-F_{A}\right)}{m}=\left(\frac{\rho_{1}}{\rho_{2}}-1\right) g .
$$

Let us assume that the vertical component of the initial velocity of the bubble is zero (the bubble is pressed to the bottom volume by the vortex roll).

Then the time of uniformly accelerated motion for elevation from height $h=0$ to height $h^{*}=H / 2$ is

$$
\tau_{a}=\sqrt{\frac{2 h^{*}}{a}}=\sqrt{\frac{2 h^{*}}{\left(\rho_{1} / \rho_{2}-1\right) g}} \approx \sqrt{\frac{H}{g}} \sqrt{\frac{\rho_{2}}{\rho_{1}}},
$$

since the gas density is much less smaller than the liquid one, $\rho_{2}<<\rho_{1}$.

For $H=5 \mathrm{~cm}$ characteristic time of the "Archimedes" floating can be calculated by formula $\tau_{a} \propto \sqrt{H / g} \sqrt{\rho_{2} / \rho_{1}} \approx \sqrt{5 \cdot 10^{-3}} \sqrt{\rho_{2} / \rho_{1}}$ (in seconds). Let the density of the liquid be $\rho_{1} \sim$ $3 \mathrm{~g} \cdot \mathrm{cm}^{-3}$ (melt of sapphire $\mathrm{Al}_{2} \mathrm{O}_{3}$ ) or $\rho_{1} \sim$ $4 \mathrm{~g} \cdot \mathrm{cm}^{-3}$ (the melt of garnet $\mathrm{Y}_{3} \mathrm{Al}_{5} \mathrm{O}_{12}$ ), and the density of the bubble be $\rho_{2} \sim 1.97 \cdot 10^{-3} \mathrm{~g} \cdot \mathrm{cm}^{-3}$ (carbon dioxide $\mathrm{CO}_{2}$ ).

Hence, we obtain the desired estimation

$$
\tau_{a} \sim(1.58-1.83) \cdot 10^{-3} \mathrm{~s} \propto 10 \mathrm{~ms} .
$$

As one can see, if we neglect the liquid viscosity, then the gas bubbles have to float from the bottom to the surface of the liquid (molten) with a sufficiently great rate. The floating time is about a few milliseconds. In this case, in stationary regime the bubbles distribution (or the impurity inclusions, for which the similar estimations can be pro- 
vided) of the melt height or the trapped bubble distribution of the crystal height, grown by HDC method, should be homogeneous. However, according to our experimental data, the maximum driftage and the subsequent capture of the bubbles occurs in the region between the convective vortices. This means that other important factors should play also essential role in hydrodynamic pattern of the bubble distribution. In particular, it is necessary to take into account the viscous resistance to motion of the gas bubbles or inclusions of impurity, since the viscosity of the crystal melt greatly exceeds the viscosity of usual liquid (e.g. water).

We estimate the characteristic time $\tau_{b}$ of hydrostatic floating with the forces of viscous resistance. As previously mentioned, the strength of this resistance obeys the Stokes formula [6], $F_{b}=-6 \pi \eta R v_{g}$, where $\eta$ - dynamic viscosity of the fluid, $v_{g} \equiv v-$ velocity of the bubble. The minus sign indicates that the Stokes force $F_{b}$ is always directed against the velocity of the moving body. We neglect for simplicity of estimation of curvilinear motion of the bubble. Then the force $F_{b}$ is directed vertically downwards against the Archimedes force. This corresponds to a simplified model of the bubble surfacing without twisting its trajectory. The rate of vertical ascent bubble varies according to the Newton's law $m_{2}(d v / d t)=F_{a}+\mathrm{F}_{b}=\left(\rho_{1}-\rho_{2}\right) g V(t)-6 \pi \eta R(t) \nu$, where $m_{2}=\rho_{2} V(t)$ and $V(t)=(4 \pi / 3) R^{3}(t)$, and $R=R(t)$ indicated by the bubble radius, which can be changed with the bubbles growth during the ascent.

The equilibrium condition $d v / d t=0$ corresponds to the achievement of a critical speed $v_{*}$ at which the viscous ascent:

$$
v_{*}=\frac{2\left(\rho_{1}-\rho_{2}\right)}{9} g R^{2} \approx \frac{2}{9}\left(\rho_{1} / \eta\right) g R^{2} .
$$

We estimate this rate. To melt $\mathrm{Al}_{2} \mathrm{O}_{3}$ and $\mathrm{Y}_{3} \mathrm{Al}_{5} \mathrm{O}_{12}$ viscosity of the order, where taken the Poiseuille and $1 P=1 \mathrm{~g} \cdot \mathrm{cm}^{-1} \cdot \mathrm{s}^{-1}$. In particular, for the melt of $\mathrm{Al}_{2} \mathrm{O}_{3}$ it is valid empirical formula $\eta=0.000067 \cdot \exp (21079 / T)$ of which for $T=2327^{\circ} \mathrm{K}$ we receive $\eta=0.58 P$. For the melt of $\mathrm{Y}_{3} \mathrm{Al}_{5} \mathrm{O}_{12}$ under $T=2240^{\circ} \mathrm{K}$ certain experimental value is $\eta=0.46 P$. The density of the melt is $\rho_{1} \sim(3-4) \mathrm{g} \cdot \mathrm{cm}^{-3}$.

Then formula (4), recorded in dimensionless variables for the critical velocity $\tilde{v}_{*:}$ is expressed in units $\mathrm{cm} \cdot \mathrm{s}^{-1}$, we obtain the relation:

$$
\tilde{v}_{*} \sim \tilde{R}^{2},
$$

where the numerical value of dimensionless radius $\tilde{R}$ should be expressed in centimeters. If the size does not exceed the gas nuclei $R<<10^{-2} \mathrm{~cm}(100 \mu \mathrm{m})$, the critical speed is reached at $v_{*}<<10^{-4} \mathrm{~cm} \cdot \mathrm{s}^{-1}$, which is very small compared with all other typical speeds in the hydrodynamic system.

The characteristic time of the order will be viscous ascent

$$
\tau_{b}(\mathrm{~cm} / \mathrm{s}) \propto \frac{2.5}{R(\mathrm{~cm})^{2}} .
$$

For the bubble of radius $R=(1-5) \cdot 10^{-3} \mathrm{~cm}$ here we get $\tau_{b} \propto\left(10^{5}-10^{6}\right) \mathrm{s} \propto 10^{2}$ hours, i.e. the free float would have to continue several days. Practically, this means that the movement in the vertical direction (the bubble floating) is going at a constant and very small velocity (less than (at most) tens of microns per second) compared with the hydrodynamic motion of the bubble (about several millimeters or centimeters per second). In such a case, the gas bubbles are nucleate on the bottom surface of the cavity, are captured by the vortex roller and carry out of them in an intermediate layer between the convective rollers located approximately at half-height of the cavity. Thus during this movement themselves bubbles continue to grow in size as one of the physical mechanisms of nucleation. This mechanism for removal of the gas bubbles inside the intermediate layer between the rollers is the initial cause of the following capture and characteristic nonuniform by the height profile of gas pores in the crystals growing by the HDC.

We estimate the characteristic time $\tau_{c}$ of hydrodynamic displacement of the bubbles that are involved by hydrodynamic flow. Such movement occurs similarly to if the bubbles were "frozen" in the line of the vortex flow and moving along them with a fluid flow during convection. Obviously, if here we ignore the viscous resistance, the characteristic velocity of the bubble would be the order of the characteristic velocity of the hydrodynamic flow in the place where situated the bubbles. In estimation the time of hydrostatic floating of bubbles it is assumed that the movement in horizontal direction is, as it was at the same velocity (uniform motion). Of course, this is strong 
approximation. In fact, the gas bubble is drawn in a circular or geometrically more complex motion of the vortex. When the lateral dimensions of the vortex are of the same order of magnitude, it can be considered that the convection vortex formed is cylindrical with the circular cross section. This is movement occurred with a constant angular velocity $\omega$ at a given distance $r$ from the axis of the vortex. In this bubble the usual centripetal (be in force) force acts with acceleration $a_{c}=\omega^{2} r$, and trajectory of the bubble in the cross section of the vortex is circular. If the bubble is emerging due to buoyancy force, its path becomes different from a circular (or more exactly, the trajectory of the vortex flow). As a result, the distance from the bubble to the axis of the vortex changes, and with it the centripetal force and the respective component of the velocity of the bubble change. But, if the change of centripetal acceleration is slow enough, i.e., radial displacement of the bubble, which trapped vortex, is small compared to the vertical displacement (ascent by the Archimedes force), the said trajectory complication bubble can be neglected. The characteristic time of the hydrodynamic motion (lift by circular rotation) of the bubble, trapped with liquid vortex, is defined as a half- period of the vortex, i.e, $\tau_{c}=\pi / \omega$, where $\omega=v / r$ - angular velocity of rotation; $v$ - regular velocity that can be taken as an average or typical fluid velocity $v^{*}$ and is typically of the order in the experiment $v^{*} \sim(1-5) \mathrm{cm} \cdot \mathrm{s}^{-1} ; r-$ rotation radius (distance from the axis of the vortex), which is an order of magnitude $r \sim H / 4$ for the case where the melt is placed only two convection rollers (height of the melt is equal to twice of diameter of the vortex).

Then

$$
\tau_{c} \sim \frac{H}{v^{*}}
$$

In the case $H \sim 5 \mathrm{~cm}$ we get an upper estimation

$$
\tau_{c} \propto 10^{1} s
$$

i.e. time of vortex removal of the bubble is in the range from seconds to fractions of a minute.

Evaluation (7) can be taken in the case when the vortex section differs from circular, e.g., when the longitudinal dimension $L$ in the direction of pulling crystal is much greater than the height $H$. At the same time the velocity of the "Archimedes" sur- facing bubble for such a system will be of the order $\tau_{a} \propto 10^{-2} \mathrm{~s}$ in the absence of viscosity and order $\tau_{b} \propto 10^{5} \mathrm{~s}$ taking into account the latest. Note that it is always very good, up to two or three orders of magnitude, the relation of the characteristic times:

$$
\tau_{a} \sim 10^{-2} \mathrm{~s}<<\tau_{c} \sim 10^{1} \mathrm{~s}<<\tau_{b} \sim 10^{4} \mathrm{~s} .
$$

The first one ensures that the dissipation-free ascent of the bubble, it almost does not feel the effect of convection. The second, on the contrary, it speaks about the near "freezing" of the trajectories of bubbles in the line of vortex flow in the presence of strong viscosity. That second condition $\tau_{b}>>\tau_{c}$ is a necessary condition for the formation of the hydrodynamic structure of gas pores. Returning to arbitrary parameters of the system, we write down the general expression for the characteristic time scale

$$
\begin{gathered}
\tau_{a} \approx\left(\frac{1}{2} g\right)^{-1 / 2}\left(\frac{H}{n}\right)^{1 / 2}\left|\frac{\rho_{2}}{\rho_{1}-\rho_{2}}\right|^{1 / 2} ; \\
\tau_{b} \approx\left(\frac{2}{9} g\right)^{-1}\left(\frac{H}{n}\right)\left(\frac{v}{R^{2}}\right)\left|\frac{\rho_{1}}{\rho_{1}-\rho_{2}}\right|^{-1} ; \\
\tau_{c} \approx\left(\frac{\pi}{2}\right)\left(\frac{H}{n}\right)\left(\frac{1}{v^{*}}\right),
\end{gathered}
$$

where indicated by the number $n$ of convective rolls that fit in the vertical direction within the height $H$ and the kinematic viscosity of the fluid $v=\eta / \rho_{1}$.

The absolute sign $|. .$.$| indicates the unit$ taking the absolute value.

The necessary condition for accumulation of the inclusions and formation of inhomogeneous spatial structure of inclusions, having the density $\rho_{2}$ and the characteristic size (radius) $R$ in the case of gas pores, and in the case of impurity inclusions, will form

$$
\tau_{b} \gg \tau_{c} \rightarrow v^{*} \gg(g / 9)\left(\pi R^{2}\right) \frac{\left|\rho_{1}-\rho_{2}\right|}{\eta} .
$$

Interestingly enough, what in this condition it does not include the geometrical dimensions of the system, and includes only the hydrodynamic parameters (velocity, density, viscosity) and the size of the inclusions.

For the gas pores with $\rho_{1}>\rho_{2}$ this condition is reduced to the restriction on the characteristic velocity $v^{*}$ of hydrodynamic flows in which dominates the removal of bubbles is convective rolls: 


$$
\tilde{v}^{*}(\mathrm{~cm} / \mathrm{s})>\left(\frac{\pi \tilde{R}^{2}}{\tilde{v}}\right) \cdot 10^{-2},
$$

wherein the values indicated tilde written in dimensionless form, the radius $\tilde{R}$ must be expressed in centimeters [cm] and the kinematic viscosity units $[\mathrm{cm} / \mathrm{s}]=\left[P /\left(\mathrm{g} / \mathrm{cm}^{3}\right)\right]$. For micron bubbles $R \sim 10^{-4} \mathrm{~cm}$ relation (12) performed extremely well for almost any hydrodynamic flows in a sufficiently viscous liquid (melt).

For the impurity inclusions in molten refractory crystals it may perform $\rho_{1} \sim \rho_{2}$ or even $\rho_{1}<<\rho_{2}$ (for example, sapphire or garnet $\rho_{1} \sim(3-4) \mathrm{g} / \mathrm{cm}^{3}$ inclusions and molybdenum or tungsten $\left.\rho_{2} \sim(10-20) \mathrm{g} / \mathrm{cm}^{3}\right)$. Inclusions with the density $\rho_{2} \geq \rho_{1}$ would have to not to float and sink into the melt, slowly sinking to the bottom, with the viscous resistance of the medium. In this case you should use a common condition follows from (11):

$$
\left|1-\frac{\rho_{2}}{\rho_{1}}\right|<\left(\frac{\tilde{v} \cdot 10^{2}}{\pi \tilde{R}^{2}}\right) \cdot \tilde{v}^{*}(\mathrm{~cm} / \mathrm{s}),
$$

wherein the magnitude $R$ should take the characteristic dimension on or value $R$ $V^{1 / 3}$, where $V-$ typical volume of one inclusion.

Note that in the case where the density of the inclusions close to the density of the melt $\rho_{1} \approx \rho_{2}$ it does not have to wait for the condition (13). In the case $\rho_{1}<<\rho_{2}$ when the condition

$$
\frac{\rho_{2}}{\rho_{1}} \geq\left(\frac{\tilde{v} \cdot 10^{2}}{\pi \tilde{R}^{2}}\right) \cdot \tilde{v}^{*}(\mathrm{~cm} / \mathrm{s})
$$

the heterogeneous structure would be absent.

But the melt viscosity of the order $\eta \sim 10^{-1} P$ and with the density $\rho_{1} \sim 3 \mathrm{~g} / \mathrm{cm}^{3}$ and size of the inclusions $\tilde{R} \sim 10^{-3} \mathrm{~cm}$ for the condition (14) leads to a very weak restrictions

$$
\frac{\rho_{2}}{\rho_{1}} \geq 10^{7} \cdot \tilde{v}^{*}(\mathrm{~cm} / \mathrm{s}),
$$

which is not realized in the experiment, even with very slow convection at velocity's $\tilde{v}^{*}$ of the order of several millimeters per minute. For disorders of forming inclusions inhomogeneous structure distribution is necessary that the size of the inclusions was more about a millimeter $R \sim 10^{-1} \mathrm{~mm}$, and the melt viscosity was very low before $\eta \sim 10^{-3} P$.
Thus, we can make a clear conclusion that for the micron inclusions in the reasonably viscous melt their heterogeneous structure is almost always obtain.

\section{Comparison of the theory with experimental data}

The resulting picture of "freezing" of the gas bubbles and microinclusions in the line of hydrodynamic flow explains the appearance of crystal zones with bad optical properties. These zones can be precisely located exactly in the intermediate layer and decorate the boundary between convection rollers. If we assume that the gas nucleus or impurity inclusions are formed in a thin zone near the lower and upper limits of the molten volume, that subsequent bubbles and inclusions are carried by hydrodynamic flow and accumulated in the zone between the convective rolls and the crystallization front. Form layer replicates geometry the surface touch the convective rolls (Fig. 1 and 2). Zone of inclusions concentration can be slightly wider due to influence of other mechanisms of removal of bubbles (e.g., buoyancy force with accounting the viscosity), which change almost "frozen" trajectory inclusion, shifting it away from the specified line of the hydrodynamic motion. However, over time, the dynamic equilibrium establishes, i.e. inclusions and come, and go from the area between the rollers, but there is some balance and there is characteristic distribution of the bubbles in the melt, and subsequently captured by the crystal gas pores (Fig. 1b).

Moreover, the bubbles of the intermediate layer can be trapped in the upper roller and move toward the free surface of the melt, thus widening in size in comparison with bubbles near the bottom (lowest size) in the intermediate layer. Therefore, the upper limit of the melt may form the zone enriched by the gas bubbles of the largest size. With such a mechanism in the crystal regions in an area between rollers, in the zone of nucleation of the bubbles (at the bottom or near the surface) and in the region of removal of the bubbles on the surface of the melt accumulation of the gas pores is maximized. Important condition for formation of such a heterogeneous distribution of the inclusions in the structure of the crystal pulled is the condition that velocity of pulling is less than velocity of bubbles removal in the intermediate layer and the velocity of setting of the equilibrium distribution. Indeed, taking it for specific times 
we get $t_{0}=L / v_{0}<<\tau_{c}$, where $t_{0}$ and $v_{0}-$ the characteristic time and the velocity of crystal pulling, and $L-$ characteristic longitudinal size of the convective roller.

Typically, $L \sim 10^{1} \mathrm{~cm}$ for the pulling velocity with (8) we obtain the relation

$$
v_{0}<<L / \tau_{c} \propto 1 \mathrm{~cm} / \mathrm{s} \propto 10^{4} \mathrm{~mm} / \mathrm{h} .
$$

It is performed with a large margin in practice.

\section{Conclusions}

Analysis of inclusions dynamics in the melt flow showed a significant effect of hydrodynamic flows in the capture and subsequent formation of the inhomogeneous distribution of inclusions in the crystals. Emerging defective areas reflect not only the unfavorable conditions at the crystallization front, but also provide important information on the hydrodynamic conditions in the melt, which should be optimized during the crystal growth.
Acknowledgement. The authors are grateful to Prof. V.V.Yanovsky for useful advice and valuable comments in the performance of this work.

\section{References}

1. Kh.S.Bagdasarov, High-temperature Crystallization from Melt, Fizmatlit, Moscow (2004) [in Russian].

2. E.R.Dobrovinskaya, L.A.Lytvynov, V.Pishchik, Sapphire. Material, Manufacturing, Applications, Springer Science+Business Media, LLC (2009).

3. A.Ja.Dan'ko, S.V.Nizhankovskiy, V.M.Puzikov et al., Crystallography Reports, 53, 1272 (2008).

4. S.V.Nizhankovskyi, A.V.Tan'ko, N.S.Sidelnikova, G.T.Adonkin, Cryst.Res.Technol., 50, 223 (2015).

5. V.G.Levich, Physicochemical Hydrodynamics, Englewood Cliffs, Prentice-Hall, N.J., (1962).

6. L.D.Landau, E.M.Lifshitz, Fluid Mechanics, vol.6 (2nd ed.), Butterworth-Heinemann (1987). 